\title{
Antioxidant enzymes in skeletal muscle and their modulation by alcohol and the dehydrogenase inhibitors 4-methylpyrazole and cyanamide
}

\author{
N. Sumien ${ }^{1}$, J. Crous ${ }^{1}$, J. A. Fegredo ${ }^{2}$ and V. R. Preedy ${ }^{2}$ \\ ${ }^{1}$ Department of Pharmacology and Neuroscience, University of North Texas Health Science Center, Fort Worth, \\ TX 76107 USA and ${ }^{2}$ Nutritional Sciences Division, King's College London, London SE1 9NU, UK
}

High concentrations of alcohol have been shown to adversely affect the metabolism of skeletal muscle but studies into antioxidant status are poorly characterised. We hypothesised that a single exposure to alcohol will impair the antioxidant status of skeletal muscle. We also hypothesised that alcohol-induced defects in muscle will be (i) prevented by 4-methylpyrazole (an inhibitor of alcohol oxidation and thus reduces acetaldehyde formation) and (ii) exacerbated by cyanamide (an inhibitor of acetaldehyde oxidation and thus increases acetaldehyde levels). To test this we used a well-validated rat model of acute alcohol exposure, and then measured total antioxidant capacity of muscle with the Oxygen Radical Absorbance Capacity (ORAC) assay and specific activities of catalase and superoxide dismutase (SOD). We used six groups of rats in the following design. Rats were dosed with a single "pre-treatment" of either saline (control), cyanamide (Cyn; $0.05 \mathrm{mmol} / \mathrm{kg} \mathrm{BW}$ ) or 4-methylpyrazole (4MP; $0.05 \mathrm{mmol} / \mathrm{kg} \mathrm{BW})$. After 30 min animals were treated with a single bolus of either saline $(0.15 \mathrm{~mol} / \mathrm{l} \mathrm{NaCl}$, controls) or ethanol $(\mathrm{EtOH} ; 75 \mathrm{mmol} / \mathrm{kg} \mathrm{BW})$ then killed $24 \mathrm{~h}$ later. The gastrocnemius muscle was dissected and used for analysis. Data were analysed with two-way ANOVA using SPSS v15.

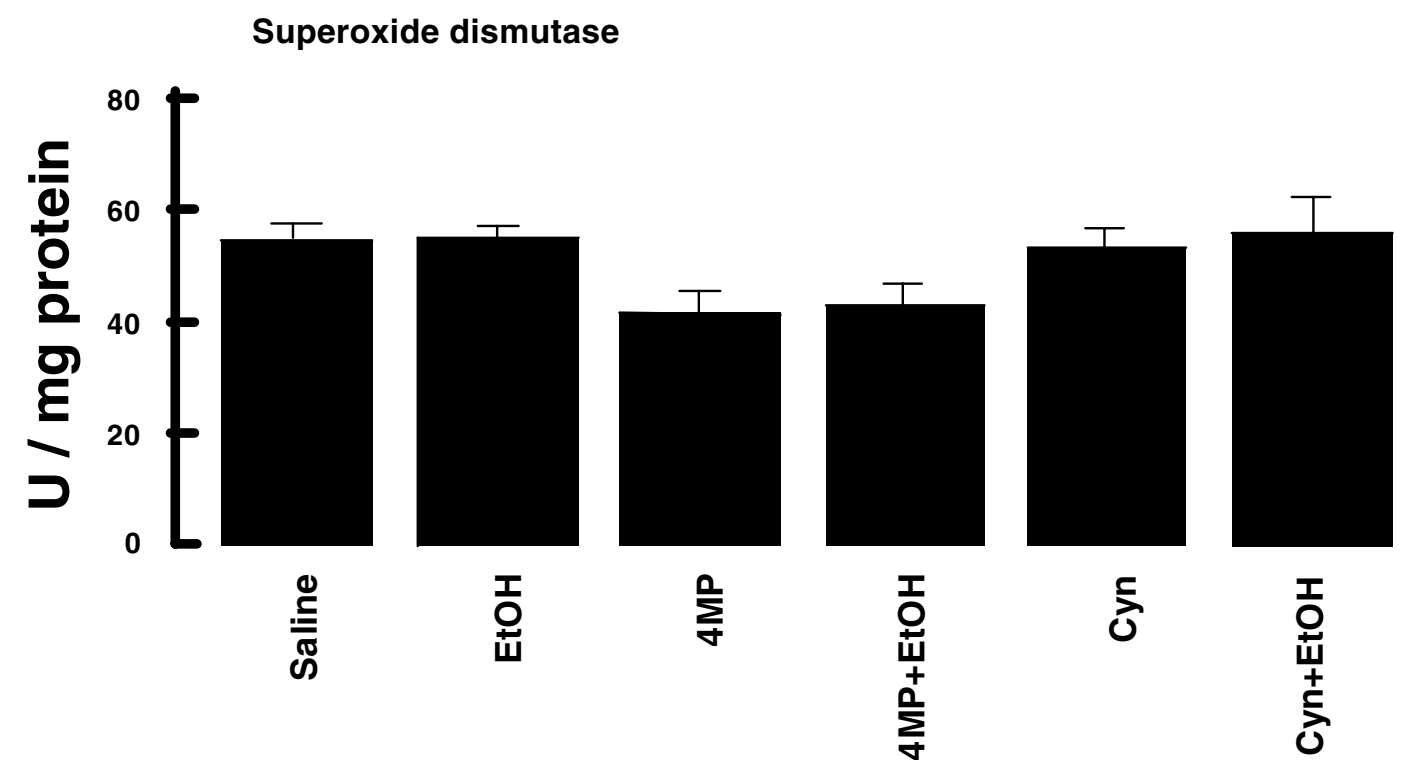

Fig. 1. Effects of ethanol on the activity of the antioxidant enzyme superoxide dismutase in the gastrocnemius muscle. Data are shown as means \pm SEM and analysed by two-way ANOVA. Pre-treatment, $P=0.02$; treatment, $P>0.05$, NS; interaction, $P>0.05$, NS.

The data showed the ORAC values and catalase activities were not overtly altered by any of the pre-treatments or treatments (data not presented for brevity). On the other hand, SOD activities were reduced by 4MP alone (Fig. 1).

In conclusion, (i) there is no evidence that alcohol per se impairs the antioxidant status of skeletal muscle and (ii) muscle dehydrogenases may play a constitutive role in maintaining the antioxidant status of skeletal muscle. 